\title{
TREATMENT OF PERFORATING ULCER OF THE FOOT
}

\author{
E. MUIR, I.L.D., M.D., M.R.C.S.E.
}

Much has been written on this subject, and the writer's apology for adding to the literature is that he has recently had considerable experience of this condition which is among the three most common and important complications of leprosy.

Definition.

Perforating ulcer differs from the other form of ulcer found in leprosy, commonly called "lepromatous": (a) in not being due to the local presence of $M$. lepræ, (b) in being a sequela of impaired trophic and sensory supply to the foot, or the part of the foot involved, (c) in being confined to the sole of the foot, while lepromatous ulcers are found on other parts of the body surface. 


\section{IODISED}

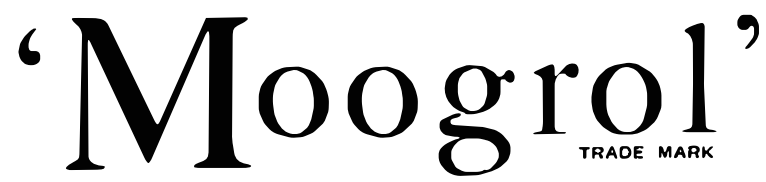

A Mixture of Esters of Acids of the Chaulmoogric Series with 0.5 per cent of Iodine

For the treatment of all forms of leprosy by combined intramuscular and intradermal injection.

The addition of iodine to the esters minimises local irritation and permits systematic infiltration of lepromata.

\section{BURROUGHS WELLCOME \& CO}

(The Wellcome Foundation Ltd)

\section{INJECTION INSTRUMENTS}

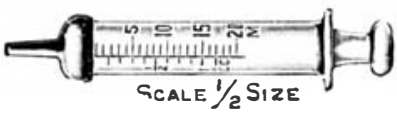

Glascord Syringes complete in case with 2 needles, in following sizes:-

20 min. and 1 c.c. $2 c . c ., 5 c . c$. , 10c.c. and 20c.c.

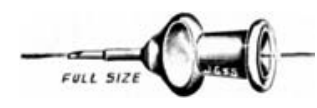

Record Needles for INTRADERMAL INJECTIONS. as illustrated, stainless steel.

Sizes 23 and 25 S.W.G.

\section{J. GARDNER \& SON}

\section{Surgical Instrument Makers} to the Edinburgh Royal Infirmary, etc.

\section{FORREST ROAD, EDINBURGH}

Works: 90 CANDLEMAKER ROW, EDINBURGH 


\section{Causation.}

The destruction or blocking of the sensory nerves, primarily by cellular pressure on, and later by fibrous constriction of, the axis cylinders deprives the foot of sensation and trophic supply and to a certain extent involves the vascular supply. The motor nerve supply is also affected when the disease spreads to the larger mixed nerves.

It is difficult to dissociate these four elements. We are all familiar with the temporary feeling of paralysis of the lower lip when an anæsthetic has been injected for extraction of a tooth from the lower jaw. By comparison it is easy to understand the paresis of the small muscles of the face, hand and foot when leprosy causes anæsthesia of the covering skin, an anæsthesia which lasts not for a few hours as in the dental extraction, but for months and years. Thus the small muscles of the foot, deprived of their normal sensory stimulus, become paretic, and fibrous tissue takes the place of muscle fibre.

These muscles normally support the arches of the foot and act as padding between the bones and the ground. They give elasticity to the foot and their tension keeps the bones of the foot strong and hard. All these functions are impaired by anæsthesia of the foot, and the muscles are further affected by interference with the motor supply; and all the parts, skin, joints and muscles, are further weakened by interference with the trophic and vascular supply.

In this weakened condition of the foot a small injury is sufficient to initiate what is known as a trophic or perforating ulcer. Such injuries are the more liable to occur as the protective influence of sensation is withdrawn, and the patient may be unaware of an abrasion caused by rubbing of the shoe or the perforation of a sharp stone, thorn or nail.

The ulcer may at first be superficial, but if not attended to at once it tends to affect the deeper structures and penetrate to the bone. Septic infection complicates the process; the soft and decalcified bone becomes carious, and an abscess of the foot may occur which, after a few days, may be discharged through the perforation.

\section{Treatment.}

Perforating ulcers may be divided into two groups: superficial and deep, the bone being affected in the latter.

Superficial ulcers generally show the qualities common to all chronic ulcers : a septic floor surrounded with raised, schlerosed non-vascular edges. Demobilisation with the application of the 
usual poultices and antiseptic dressings may cause healing, and this process may be accelerated by infiltration into the surrounding subcutaneous tissue of a mildly ir ritant fluid, such as hydnocarpus oil ( 1 or 2 c.c) or a 1 in 4 solution of Dettol ( 1 to 2 c.c), which causes exfoliation of the thick surrounding cuticle.

When the bone is involved its removal is essential, and it will be found wise to reverse the usual surgical rule and not be too conservative in the amount removed.

While perforating ulcers may occur anywhere in the sole of the foot, by far the commonest sites are the heads of the metatarsals, the first metatarsal being that most commonly involved.

II'hile a more conservative operation may suffice in the slighter cases, the operation of choice is complete metatarsectomy. This causes narrowing of the foot, but the result is much more likely to be permanent. There is nothing more damaging to the health and confidence of the patient than an ulcer which recurs whenever he begins to walk again.

\section{Metatarsectomy.}

The writer recommends the following technique which he has found simple, rapid and satisfactory in its results. An anæsthetic is seldom necessary as sensation is almost entirely absent. The patient lies on the table with his foot projecting and the end of the table supporting his tendo archilles. After the parts have been thoroughly cleaned and iodine has been applied, a tourniquet is applied firmly over a piece of lint round the middle of the thigh. Sterile towels are arranged so that only the distal part of the foot is uncovered. An incision is made in the sole beginning at the ulcer, extending for the whole length of the metatarsus and cutting down to the bone. The bone is quickly cleared, as is also the proximal phalanx if it is involved. A small cutting bone forceps is useful in disarticulating the bone and disengaging it from its bed. When the bone has been removed the sides of the ulcer are dissected out and the wound is trimmed, the edges being undercut and freed so that they come together, if possible, without tension. Sulphanilamide powder is rubbed thoroughly into the wound. Sterile gauze is inserted sufficient to stop bleeding but not to prevent the approximation of the edges; the end of the gauze projects through the dependent end of the wound. The wound is closed with a few strong, deep sutures, and over an outside dressing a tight bandage is applied. The tight packing of gauze, the sutures and the tight bandage do away with the necessity of applying ligatures, thus saving much time. The whole operation may be finished in 15 
to 20 minutes, and this is important, as the patient generally begins after that time to complain of the pressure of the tourniquet. This is removed as soon as the first few turns of the bandage have been made. The sulphanilamide powder keeps the wound clean, so that the first change of dressing can be delayed for forty-eight hours, by which time the danger of bleeding is past. The gauze is removed and a thin wick of gauze powdered with sulphanilamide powder inserted.

The wound generally takes about three to four weeks to heal, but the patient should not apply his foot to the ground for a further four weeks, so as to give the fibrous tissue time to consolidate.

After the first forty-eight hours the patient should be encouraged to walk about on crutches, keeping his foot carefully off the ground.

The last two rules are indeed important in the treatment of all perforating ulcers even when an operations has not been necessary.

When a perforating ulcer occurs, as is less often the case, in connection with the heel, operative measures are less satisfactory. If rest, dressings, and infiltration of the subcutaneous tissue round the ulcer do not bring about a permanent cure, a layer of the bone in the base of the wound may be gouged out and the edges undermined, trimmed and brought together as described in the operation above.

After operation, or in simple cases where operation is not necessary, unless the healed wound has time to consolidate (generally 3 to 4 weeks), it is likely to recur; but the use of crutches removes the need for confinement to bed, which is so harmful to the general health and interferes with the beneficial effects of exercise in the treatment of leprosy.

In many cases the writer has found the progress of the patient handicapped, or altogether stopped, by the demobilisation attendant on perforating ulcers as well as by septic absorption from the wound. On this account he considers it imperative that immediate steps should be taken to effect permanent healing. Much can be done to prevent the occurrence of perforating ulcer by the use of proper footwear, the application of suitable padding inside the shoes, and careful hygiene of the feet. When anæsthesia of the feet occurs the patient should be warned beforehand of the dangers and taught how to avoid them. 